'Departamento de Neurociencia. Universidad de Chile. Santiago, Chile.

${ }^{2}$ Programa de Neurología, Facultad de Ciencias Médicas, Universidad de Santiago de Chile. Santiago, Chile.

${ }^{3}$ Departamento de Neurología y Neurocirugía, Hospital Clínico de la Universidad de Chile. Santiago, Chile.

${ }^{4}$ Departamento de Otorrinolaringología, Hospital Clínico de la Universidad de Chile. Santiago, Chile.

Financiamiento: C.D. es financiada por proyecto Anillo ACT1403, CONICYT, PIA. P.H.D. es financiado por Fondecyt 1161155, CONICYT BASAL FB008 y Proyecto ICM P09-015F.

Los autores declaran no tener conflictos de interés.

Recibido el 9 de abril de 2019, aceptado el 23 de marzo de 2020.

Correspondencia a: Dr. Paul Délano Reyes Santos Dumont 999, Departamento de Otorrinolaringología, Hospital Clínico de la Universidad de Chile. Santiago, Chile. 8380456 Teléfono: +562 29788153 pdelano@med.uchile.cl

\section{Hipoacusia como factor de riesgo para demencia}

\author{
JONATHAN WIMMER DEL SOLAR ${ }^{1,2}$, CAROLINA DELGADO ${ }^{1,3}$, \\ MARIELA C. TORRENTE ${ }^{4}$, PAUL H. DÉLANO ${ }^{1,4}$
}

\section{Hearing loss as a risk factor for dementia}

In the last decades, there has been an increase in life expectancy in the world, with the consequent modification in the proportion of adults over 60 years. This is accompanied by an increase in pathologies for which aging is the main risk factor, such as dementia and hearing loss, which profoundly affect the quality of life of individuals and their family and impact health system costs. Given the lack of disease-modifying treatments for dementia, the study of mechanisms to prevent its occurrence has become a world priority. In the year 2017, the Lancet "Commission for dementia prevention, intervention, and care" proposed a model, in which hearing loss emerged as a new modifiable risk factor for the development of dementia. This result undoubtedly has important consequences for the understanding the multifactorial nature of dementia, our daily clinical practice and public policies aimed at its prevention and treatment. In this article, we review the current evidence supporting the association between dementia and hearing loss, discussing the available strategies for prevention, detection and treatment of hearing loss and its possible impact on the natural course of dementia. A flow chart for the clinical management of different subgroups of patients is proposed.

(Rev Med Chile 2020; 148: 1128-1138)

Key words: Aging; Cognitive Dysfunction; Dementia; Hearing Loss; Presbycusis.
Envejecimiento poblacional, demencia e hipoacusia

egún la Organización Panamericana de la Salud, la expectativa de vida al nacer en Chile ha ido en aumento, siendo actualmente de 80 años para los hombres y 85 años para las mujeres, con lo cual la proporción de personas que sobrepasa los 60 años ya alcanzaba $14 \%$ en el año $2014^{1}$. A nivel mundial se observa la misma tendencia, con $12 \%$ de las personas mayores a 60 años en la actualidad y una proyección de $21,5 \%$ para el año $2050^{2}$.

Estos cambios demográficos se acompañan de un aumento en la prevalencia de patologías asociadas a la edad, como la demencia y la hipoacusia. A nivel global, la demencia se ha convertido en un problema prioritario de salud pública, estimándose una prevalencia mundial de 50 millones de personas en el año $2017^{3}$, cifra que aumentaría a más del doble para el año 2050, afectando principalmente a países de ingresos bajos o medios, como es el caso de los países latinoaméricanos ${ }^{4}$.

En Chile, según el Estudio Nacional de la Dependencia del Servicio Nacional del Adulto Mayor, SENAMA 2009, 7,1\% de las personas mayores de 60 años padece algún tipo de demencia ${ }^{5}$. Los altos costos personales y sociales de padecer una demencia ${ }^{6-9}$, asociado a la falta de terapias que modifiquen el curso de la enfermedad, ha hecho de manifiesto la importancia de comprender los factores de riesgo que determinan su desarrollo con el fin de prevenir o modificar su curso. Se ha 
estimado que el retardo en el inicio de la demencia en 5 años disminuiría la prevalencia de esta en alrededor de $50 \%{ }^{10}$.

Según un trabajo de consenso publicado en la revista Lancet $^{11}, 35 \%$ del total de los factores de riesgo que influyen en el desarrollo de una demencia son modificables, incluyendo: nivel educacional, hipertensión arterial, diabetes mellitus, obesidad, tabaquismo, sedentarismo, depresión, aislamiento social y la pérdida auditiva. En este modelo emergen la hipoacusia y el aislamiento social como nuevos factores de riesgo modificables. Más aún, la alta prevalencia de hipoacusia y su elevado riesgo relativo (1,9 RR), la convierten en el principal factor de riesgo modificable para el desarrollo de una demencia y en un interesante blanco terapéutico.

En este artículo, revisaremos la evidencia actual que sustenta esta asociación, discutiendo las estrategias disponibles de prevención, detección y tratamiento para hipoacusia, contextualizándola en relación a su posible impacto en el curso natural de la demencia, proponiendo un flujograma de estudio para diferentes subgrupos de pacientes, complementando la información de las actuales guías GES de Hipoacusia en mayores de 65 años, y el plan Nacional de Demencia. Cabe destacar que, al momento de la formulación de este manuscrito, aún no se han publicado las guías GES de enfermedad de Alzheimer y otras demencias.

\section{$\underline{\text { Hipoacusia: definición, causas y epidemiología }}$}

Según la Organización Mundial de la Salud, la hipoacusia se define como una disminución del promedio total puro de las frecuencias de 0,5 a 4 $\mathrm{kHz}$ mayor a $25 \mathrm{~dB}$ HL en el mejor oído, medido con audiometría tonal ${ }^{12}$, ya que desde este nivel existe una alteración en la comprensión del habla en situaciones con ruido ambiental, afectando la comunicación oral. Se clasifica en hipoacusia leve (25 a $40 \mathrm{~dB} \mathrm{HL})$, moderada (41 a $60 \mathrm{~dB} \mathrm{HL})$, severa (61 a $80 \mathrm{~dB} H \mathrm{HL}$ ) y profunda (mayor a 81 dB HL). Se estima que la hipoacusia afecta a $6,1 \%$ de la población mundial, y se espera que siga aumentando en las próximas décadas, sobrepasando la cifra de 1.000 millones de personas para el año $2025^{13}$. Al igual que la demencia, su prevalencia aumenta con la edad ${ }^{14-16}$, alcanzando hasta $44,3 \%$ de las personas mayores de 60 años ${ }^{14}$. En Chile, $52 \%$ de las personas sobre 65 años reporta tener problemas de audición ${ }^{5}$.

Las causas de hipoacusia son múltiples. Puede tener su origen en patología del conducto auditivo externo (por ejemplo, tapón de cerumen), oído medio (por ejemplo, otitis media crónica), u oído interno (por ejemplo, exposición laboral a ruido). En la población de adultos mayores la "presbiacusia" o pérdida auditiva relacionada al envejecimiento destaca como la alteración sensorial más frecuente, caracterizándose por una pérdida auditiva bilateral progresiva, con problemas para la comprensión del lenguaje hablado, especialmente en ambientes ruidosos ${ }^{17,18}$.

\section{Asociación entre hipoacusia y demencia}

Estudios epidemiológicos recientes han hecho evidente la asociación entre la disfunción sensorial y deterioro cognitivo en adultos mayores, tanto en personas con problemas visuales ${ }^{19-21}$ como con hipoacusia. Esta última condición es la que cuenta con un mayor número de estudios, mostrando una asociación independiente (ajustada por edad $\mathrm{y}$ factores de riesgo cardiovascular) con deterioro cognitivo en adultos mayores ${ }^{11,22-30}$, afectando la rapidez de procesamiento cognitivo, funciones ejecutivas, memoria y estatus cognitivo global ${ }^{31,32}$. En el metaanálisis realizado por Livingston y cols. publicado en Lancet $2017^{11}$, se incluyeron 3 estudios prospectivos con seguimientos entre 9 y 17 $\operatorname{años}^{22,23,27}$, encontrando un RR $>1,94$ de desarrollar demencia en personas con deterioro auditivo, con una relación dosis respuesta relativa al grado de hipoacusia (RR 1,89, 3,00 y 4,94 en hipoacusia leve, moderada y severa $)^{27}$.

Los mecanismos fisiopatológicos que explican esta asociación son motivo de discusión ${ }^{33,34}$. El envejecimiento sería un factor común entre ambas condiciones, con tres mecanismos interrelacionados que llevarían al deterioro cognitivo como consecuencia de una hipoacusia: (i) un aumento de la carga cognitiva impuesta a las personas con hipoacusia para comprender lo que oyen, requiriendo la utilización de otros dominios cognitivos como mecanismo compensatorio ${ }^{35}$; (ii) cambios estructurales y funcionales en sujetos con hipoacusia, tanto a nivel de la cóclea y del nervio auditivo ${ }^{18}$ como a nivel del sistema nervioso cen- 
tral en regiones auditivas ${ }^{36-42} \mathrm{y}$ áreas relacionadas directamente con otros dominios cognitivos ${ }^{35,43}$; (iii) aislamiento social secundario a las dificultades comunicacionales impuestas por la hipoacusia, lo que podría agravar o facilitar el desarrollo de una demencia ${ }^{11,33,44-48}$.

Además del aislamiento social, la hipoacusia se asocia a trastornos ansiosos y depresión ${ }^{30,49,50}$, los que también influirían en el funcionamiento cognitivo del adulto mayor. Si bien existe discusión acerca de si la hipoacusia afecta directamente la cognición o lo hace secundariamente a través de estos otros factores, variados estudios sugieren su relación causal directa, ya que tanto en modelos animales ${ }^{51,52}$ como en seres humanos ${ }^{53}$ la hipoacusia produce cambios en múltiples áreas cerebrales. Sumado a esto, en estudios de cohorte se observa que la hipoacusia precede a la demencia por más de 9 años ${ }^{22,23,27,54}$, presentando una relación entre grado de hipoacusia y riesgo de desarrollar una demencia $^{27}$, mostrando efectos independientes de la hipoacusia y estos otros factores sobre la cognición ${ }^{44}$.

\section{Hipoacusia y demencia: prevención, detección y tratamiento}

Con la nueva evidencia disponible, cómo enfrentamos la pregunta de: ¿Qué puedo hacer para prevenir o retardar el inicio de una demencia? Generalmente, nuestra primera aproximación es prevenir y tratar factores de riesgo cardiovascular $^{55,56}$, practicar ejercicio físico regularmente y mantener una alimentación equilibrada ${ }^{57}$. La evidencia actual indica que, de todos los factores de riesgo modificables para el desarrollo de una demencia, la hipoacusia es la que tiene un mayor peso relativo ${ }^{11}$, observándose sobre todo en casos con pérdida auditiva $>40 \mathrm{~dB}^{22}$. Por lo tanto, en la actualidad se hace indispensable la promoción de medidas de prevención de la hipoacusia a lo largo de la vida, incluyendo su detección y tratamiento, así como también, la necesidad de realizar estudios que demuestren la eficacia de estas intervenciones, ya que aún no está claro el impacto de la prevención y tratamiento de la hipoacusia en el desarrollo de una demencia. En la Figura 1 se muestra el esquema de estudio propuesto por los autores, el cual complementa la información de la guía clínica GES de hipoacusia del adulto mayor, contextualizando esta patología en su interrelación con la demencia.

\section{Prevención del desarrollo de la hipoacusia: Políticas públicas y el rol del personal de salud}

Se estima que cerca de $50 \%$ de los casos de hipoacusia a nivel mundial serían prevenibles a través de políticas públicas ${ }^{12}$. Dentro de estas medidas se encuentran la prevención y tratamiento de patologías infectocontagiosas a través de inmunizaciones, educación sexual y tratamiento antibiótico oportuno, la prevención de causas obstétricas y perinatales a través del control y seguimiento materno infantil ${ }^{58}$ y la implementación de políticas que favorezcan disminución del ruido ambiental y laboral ${ }^{59}$, el uso racional de medicamentos ototóxicos como aminoglucósidos ${ }^{58}$, y la prevención y el tratamiento de factores de riesgo modificables compartidos por la hipoacusia y la demencia, como la hipertensión, la diabetes y el tabaquismo, a pesar de que para estos últimos, su asociación con la hipoacusia es controvertida ${ }^{56,60,61}$ (Figura 2a).

\section{Detección de la hipoacusia}

La audiometría tonal es el método de elección para el diagnóstico de hipoacusia ${ }^{12}$. Cabe destacar que, si bien una estrategia ideal sería plantear la realización de una prueba de detección en un solo paso a través de audiometría, como ha sido sugerido por algunos autores ${ }^{62}$, en la práctica, este examen requiere de infraestructura especializada (cámara silente y audiómetro) y un profesional entrenado en el área de audiología, lo cual limita su accesibilidad en la atención primaria de salud, haciéndola menos costo-efectiva que otros métodos $^{63}$. Por esta razón se han propuesto diversos estudios de tamizaje para hipoacusia para el médico general (Figura 2b). Estas pruebas varían desde la autopercepción de hipoacusia, encuestas específicas, test del susurro, evaluaciones mediante aplicaciones de dispositivos móviles, entre $\operatorname{otros}^{64,65}$ y a pesar que en la actualidad no son recomendadas como método de tamizaje en población general ${ }^{64,66}$, si son herramientas útiles para la sospecha en subpoblaciones de riesgo, como lo son los adultos mayores ${ }^{65}$, por lo que, desde el punto de vista de los autores, en pacientes con factores de riesgo para hipoacusia y/o demencia todo médico debería realizar un test de tamizaje auditivo y, según su resultado, derivar al especialista (Figura 1). 


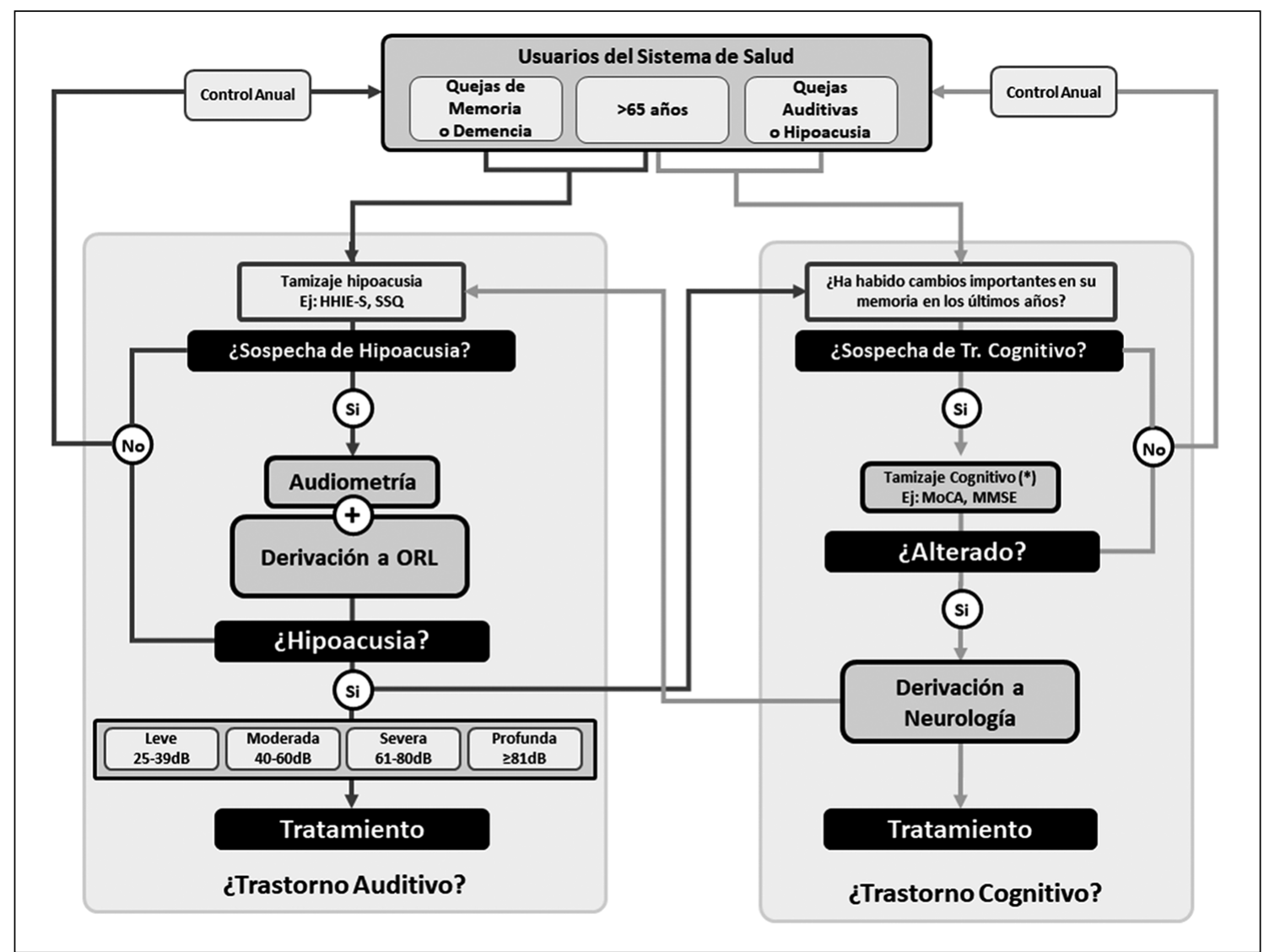

Figura 1. Flujograma para el estudio de pacientes según características demográficas y factores de riesgo. Aquellos pacientes con el diagnóstico de demencia, quejas subjetivas de memoria o todo paciente mayor a 65 años, se plantea la necesidad de realizar un de tamizaje auditivo, de resultar alterado, se debe solicitar una audiometría confirmatoria y realizar la derivación a un servicio de Audiología para evaluación por especialista. El especialista evaluará el resultado de la audiometría, clasificándola según el nivel de compromiso y realizando el tratamiento correspondiente o derivar a APS para controles periódicos en caso de resultar normal. En todo paciente mayor de 65 años o con quejas auditivas o hipoacusia, se debe realizar un primer paso con una pregunta exploratoria acerca de su estado de memoria, en caso de existir sospecha de un trastorno cognitivo, se recomienda la realización de un test de tamizaje cognitivo estandarizado, y si éste resulta alterado, entonces se debe derivar a especialista para evaluación e inicio de tratamiento. A nivel de especialista también es necesario, en el caso de los pacientes con hipoacusia como en pacientes con trastornos cognitivos, realizar estudios de tamizaje y posterior derivación en caso que no haya sido realizado previamente en APS. En pacientes menores de 65 años y sin hipoacusia o quejas de memoria se recomienda la prevención primaria de hipoacusia (Figura 2A). (APS: Atención Primaria de Salud; ORL: Otorrinolaringólogo, HHIE-S: Shortened Hearing Handicap Inventory for the Elderly, SSQ: The Speech, Spatial and Qualities of Hearing Scale, MoCA: Montreal Cognitive Assessment; MMSE: Mini-mental state examination). ${ }^{*}$ El tamizaje cognitivo debe realizarse con precaución, ya que tiene un importante sesgo sociocultural viendo afectados sus resultados en personas con baja escolaridad según lo reportado por Quiroga y cols. 2004 con el MMSE y Delgado y cols 2012 con el MOCA, recomendándose solo en pacientes con quejas de memoria, no así en pacientes asintomáticos. Por otro lado el MMSE es muy poco sensible para etapas leves o casos en que predominan la disfunción ejecutiva.

\section{Tratamiento de la hipoacusia}

Respecto al tratamiento de la hipoacusia, éste dependerá según su causa, existiendo intervenciones quirúrgicas para casos seleccionados (e.g. otoesclerosis, otitis media crónica) y diferentes alternativas de dispositivos de asistencia auditiva (DAA) dentro de los cuales destacan audífonos, implantes cocleares ${ }^{67}$ e implantes auditivos de conducción ósea ${ }^{68,69}$ (Figura 2c). En cuanto a su efectividad, existe evidencia de que aquellos 


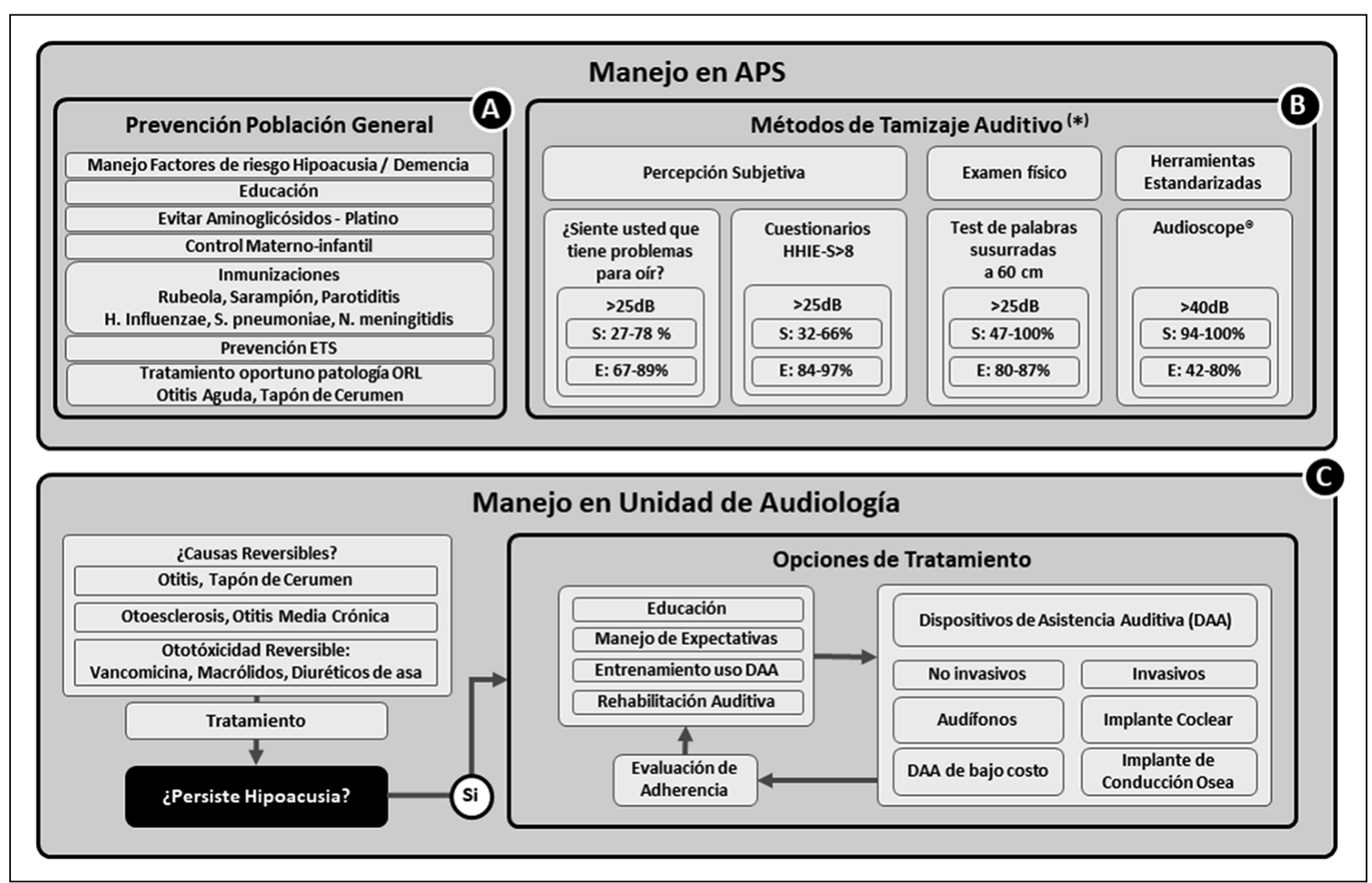

Figura 2. Esquemas de prevención, estudio y tratamiento a nivel de APS y de especialidad. (A) Estrategias para la prevención de hipoacusia. (B) Test de tamizaje auditivo con sus respectivas sensibilidades y especificidades. (C) Estudio por especialista posterior a derivación de pacientes con audiometrías compatibles con hipoacusia y estrategias de tratamiento. ${ }^{(*)}$ Sensibilidad y especificidad según Chou y cols. 2011.

pacientes que efectivamente utilizan sus audífonos tienen una mejoría en su calidad de vida, comunicación y en su funcionamiento social y emocional ${ }^{70-72}$. En Chile, el tratamiento por medio de audífonos para pacientes con hipoacusia mayores de 65 años fue incorporado como patología GES el año 2007, siendo uno de los pilares en las políticas públicas en salud auditiva del país ${ }^{73}$. A través de este programa se entrega un audífono por año a pacientes mayores de 65 años con hipoacusia al menos moderada en el mejor oído. Cabe destacar que solo se garantiza la entrega del audífono, no incluyendo la atención por médico especialista en otorrinolaringología ni examen de audiometría tonal.

A pesar de su eficacia en el tratamiento de la hipoacusia, existen dos problemas importantes en la efectividad de estos dispositivos. En primer lugar, su alto costo, haciéndolos inaccesibles para muchos usuarios $^{74}, y$, en segundo lugar la baja prevalencia de uso en aquellas personas con indi- cación de usarlos, siendo más alta mientras más profunda la hipoacusia ${ }^{65,75}$, alcanzando valores en series internacionales entre $11 \%$ y $37 \%{ }^{76-79}$. En Chile, se ha reportado una adherencia al uso de audífonos de entre $40 \%$ y $80 \%{ }^{80,81}$. Si bien la prevalencia de uso es baja, existen estudios que muestran una tendencia a un aumento de ésta en los últimos años ${ }^{76}$, sobre todo al utilizar estrategias capaces de mejorar su utilización ${ }^{81}$.

Para atacar los dos principales problemas del tratamiento con DAA, se han desarrollado DAA de bajo costo con buenas características técnicas que abren nuevas alternativas para el tratamiento de personas con hipoacusia ${ }^{82}$. Un programa piloto realizado en Baltimore, entregó DAA de bajo costo (Williams Sound Pocketalker ${ }^{\infty}$ o Sound World Solutions CS- $50^{\oplus}$ ) a adultos mayores de 60 años, asociando además intervenciones que incluían un estudio de audición, educación acerca de las características y limitaciones cada uno de los implementos, entrenamiento en su uso y entrega de material 
de rehabilitación auditiva, obteniendo resultados favorables en variables sociales y emocionales a los 3 meses $^{83,84}$, abriendo nuevas posibilidades a futuro para la accesibilidad de tratamiento auditivo para personas con demencia.

\section{Disminuir el riesgo de la demencia a través de la corrección auditiva: ¿Es una estrategia efectiva?}

En la actualidad, no está claro si el corregir la hipoacusia es una estrategia efectiva para retardar el desarrollo de una demencia. Existe abundante evidencia tanto a favor del uso de audífonos ${ }^{53,44,85-88}$ e implantes cocleares ${ }^{89}$, como evidencia en con$\operatorname{tra}^{86,75}$, siendo controvertido si la mejoría cognitiva en personas que utilizan audífonos se debe a una mejoría en variables sociales y emocionales más que a un efecto directo a nivel cognitivo. Actualmente, está en curso el primer estudio randomizado controlado multicéntrico con el objetivo de resolver estas incógnitas ${ }^{70}$, lo que será esencial para aclarar la relación causal entre hipoacusia y demencia.

\section{Hipoacusia y demencia: a quién estudiar}

En la población general de adultos mayores, no existe consenso acerca de quién, a qué edad y con qué frecuencia realizar un tamizaje auditivo. Esto ha generado un llamado de atención acerca de la necesidad de realizar estudios de alta calidad para responder estas incógnitas ${ }^{64,66,90}$.

Pese a esta falta de consenso, existen varios puntos que hacen suponer un potencial beneficio de la detección temprana de la hipoacusia ${ }^{91}$, ya que se ha descrito que desde que comienza el deterioro auditivo hasta que se busca atención especializada pasan aproximadamente $10 \mathrm{años}^{92}$, debido en parte a que una gran proporción de los pacientes con hipoacusia no son conscientes de sus problemas auditivos ${ }^{66,93}$, presentando una hipoacusia subclíni$c a$, en la que se estima que ya estarían ocurriendo cambios estructurales y funcionales en el cerebro que explicarían la asociación entre hipoacusia y demencia ${ }^{53,32,37}$, generando una ventana temporal en la que se podría intervenir.

Por estos motivos, los autores planteamos que la decisión de realizar un estudio auditivo o no a un paciente debe realizarse caso a caso. Nosotros proponemos una evaluación diferencial dependiendo del rango etario al que pertenezcan, con un punto de corte en 65 años, ya que a esta edad se garantiza la entrega de audífonos por el sistema GES, y según la presencia o no de factores de riesgo para el desarrollo de hipoacusia o demencia (Figura 1, Tabla 1).

\section{Tabla 1. Recomendaciones para prevención, detección y tratamiento de la hipoacusia y nivel de evidencia (nivel de evidencia codificado según guías de recomendación de la American Heart Association)}

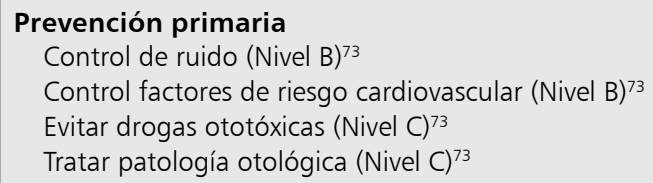

\section{Tratamiento}

Implantes cocleares (Evidencia Nivel B) ${ }^{67}$

Implantes Auditivos de conducción osea (Evidencia Nivel C) ${ }^{68}$

Audífonos (Evidencia Nivel A) ${ }^{73}$

Dispositivos de Asistencia Auditiva de Bajo Costo (Evidencia Nivel C) ${ }^{84}$

\section{Detección}

Población > 65 años

Tamizaje auditivo + Confirmación con audiometría $(\text { Evidencia Nivel } A)^{73}$

Personas con quejas de audición

Evaluar si existen quejas cognitivas (Evidencia Nivel C)

Reevaluación anual

Personas con quejas cognitivas

Tamizaje Cognitivo (Evidencia Nivel C) ${ }^{100}$

(Evidencia Nivel C) ${ }^{73}$

Tamizaje auditivo + Confirmación con audiometría (Evidencia Nivel C) $^{98}$ 


\section{Tamizaje poblacional general (menores de 65 años)}

A pesar de la alta carga de enfermedad que conlleva la hipoacusia tanto por su alta prevalencia como por las consecuencias negativas para la salud y calidad de vida, las altas tasas de falsos positivos de las pruebas de tamizaje ${ }^{64,66} \mathrm{y} \mathrm{el}$ alto costo y baja prevalencia de uso de los DAA, han llevado a que las normas internacionales no aconsejen la implementación de pruebas de tamizaje general en la población adulta ${ }^{64,66}$, por lo que, en personas menores de 65 años, nuestro deber es la prevención primaria de la hipoacusia y demencia a través del control de factores de riesgo (Figura 2a).

\section{Personas mayores de 65 años, sin quejas de memoria}

El principal factor de riesgo para el desarrollo de una demencia es la edad, contribuyendo también como un factor relevante para el desarrollo de una hipoacusia ${ }^{18}$. Por lo tanto, en la opinión de los autores, todo paciente mayor de 65 años, debería realizarse un estudio de tamizaje auditivo, utilizando cuestionarios auto contestados como las preguntas utilizadas en la encuesta nacional de salud o el hearing handicap inventory for the elderly $(\text { HHIE })^{94} u$ otros que pudiesen ser validados en la población nacional. Dejando la evaluación audiométrica solo para los que tengan un resultado alterado en el autoreporte.

\section{Personas con quejas subjetivas de memoria y deterioro cognitivo}

En pacientes que ya presentan quejas subjetivas de memoria o deterioro cognitivo, es necesario tratar los factores de riesgo que sean modificables ${ }^{95}$, por ejemplo, depresión, trastornos metabólicos, factores de riesgo cardiovascular o hipoacusia ${ }^{11}$. En el caso de la hipoacusia, se ha descrito que esta puede influir directamente en los resultados de las pruebas cognitivas utilizadas para el diagnóstico de deterioro cognitivo ${ }^{96,97}$, además de ser un factor agravante en pacientes con demencia en los casos de hipoacusia severa a profunda, facilitando la aparición de episodios de delirio intercurrentes ${ }^{11}$. Por lo tanto, nuestra recomendación en este grupo de pacientes es realizar un estudio auditivo tanto al momento del diagnóstico como de forma periódica durante su seguimiento ${ }^{98}$ (Figura 1, Tabla1).

\section{Personas con quejas auditivas o hipoacusia}

A los pacientes que consultan por quejas auditivas o problemas de comunicación, se les debe pedir una audiometría. Considerando los antecedentes expuestos a lo largo de esta revisión, en cualquier paciente que presente una hipoacusia $>40 \mathrm{~dB}$, será relevante descartar un trastorno cognitivo asociado. Por lo tanto, nuestra recomendación es explorar la existencia de problemas de memoria inicialmente a través de una pregunta general, para luego realizar una prueba de tamizaje específica solo si existen quejas cognitivas según el paciente o el acompañante ${ }^{99,100}$ (Figura 1, Tabla1), siempre teniendo en cuenta que los resultados de estas pruebas deben tomarse con precaución, ya que sus puntajes se ven influidos por variables socioculturales, como el nivel educacional del paciente ${ }^{101}$.

\section{Conclusiones}

La demencia es una patología de alta prevalencia, con consecuencias devastadoras tanto para quienes la padecen como para su entorno cercano. Con el envejecimiento poblacional se espera que su prevalencia siga en aumento. La falta de tratamientos que modifiquen el curso de esta enfermedad hace indispensable un enfoque preventivo, actuando sobre factores de riesgo modificables. En los nuevos modelos de prevención, la hipoacusia emerge como un factor relevante. La asociación epidemiológica entre hipoacusia y demencia ha sido establecida de manera independiente de la edad y los factores de riesgo cardiovasculares, a pesar de esto, el mecanismo de esta asociación es desconocido. La posibilidad de prevenir una demencia actuando sobre la hipoacusia ha abierto una prometedora línea de intervención, con aún muchas incógnitas por resolver. En el proceso de sospecha diagnóstica, estudio y tratamiento es indispensable un trabajo multidisciplinario que incluye la participación de médicos generales, médicos especialistas, fonoaudiólogos, tecnólogos médicos y otros miembros del equipo de salud.

\section{Referencias}

1. Pan American Health Organization. Health in the Americas+, 2017 Edition Summary: regional outlook and country profiles. [Internet]. Pan american Health 
Organization; 2017 [Consultado el 26 de diciembre de 2018]. Disponible en: http://iris.paho.org/xmlui/handle/123456789/34321.

2. United Nations. World Population Prospects: The 2017 Revision - Data Booklet [Internet]. Population Division. 2017 [Consultado el 28 de diciembre de 2018]. Disponible en: https://population.un.org/wpp/Publications/ Files/WPP2017_DataBooklet.pdf.

3. World Health Organization. Dementia Fact sheet. [Internet]. WHO. 2017 [Consultado el 2 de enero de 2019]. Disponible en: https://www.who.int/news-room/ fact-sheets/detail/dementia.

4. Prince M, Guerchet M, Prina M. Policy Brief: The Global Impact of Dementia 2013-2050. Alzheimer's Dis Inter [Internet]. 2013 [Consultado el 26 de diciembre de 2018]. Disponible en: https://www.alz.co.uk/research/ GlobalImpactDementia2013.pdf.

5. Ministerio de Salud [MINSAL]. Plan Nacional de Demencia. Atención Primaria. 2017; 60. Disponible en: https://www.minsal.cl/wp-content/uploads/2017/11/ PLAN-DE-DEMENCIA.pdf.

6. Cotter VT. The burden of dementia. Am J Manag Care. 2007; 13 (12) Suppl 8: S193-7.

7. Hojman DA, Duarte F, Ruiz-Tagle J, Budnich M, Delgado C, Slachevsky A. The cost of dementia in an unequal country: The case of Chile. PLoS One 2017; 12 (3): e0172204. Disponible en: http://www.ncbi.nlm.nih. gov/pubmed/28267795.

8. Vieira RT, Caixeta L, Machado S, Silva AC, Nardi AE, Arias-Carrión O, et al. Epidemiology of early-onset dementia: a review of the literature. Clin Pract Epidemiol Ment Health 2013; 9 (6): 88-95.

9. World Health Organization. Alzheimer's Disease International. Dementia: A Public Health Priority. World Heal Organ [Internet]. 2012 [Consultado el 30 de enero de 2019]. Disponible en: https://www.who.int/ mental_health/neurology/dementia/dementia_thematicbrief_executivesummary.pdf.

10. Brookmeyer R, Gray S, Kawas C. Projections of Alzheimer's disease in the United States and the public health impact of delaying disease onset. Am J Public Health 1998; 88 (9): 1337-42.

11. Livingston G, Sommerlad A, Orgeta V, Costafreda S, Huntley J, Ames D, et al. Dementia prevention, intervention, and care. Lancet (London, England) 2017; 390 (10113): 2673-734.

12. WHO Media centre. WHO | Deafness and hearing loss. World Heal Organ [Internet]. 2018 [Consultado el 30 de enero de 2019]. Disponible en: https://www.who.int/ news-room/fact-sheets/detail/deafness-and-hearing-loss

13. WHO | Estimates. WHO [Internet]. 2018 [Consultado el
26 de diciembre de 2018]; Disponible en: https://www. who.int/pbd/deafness/estimates/en/

14. Goman AM, Lin FR. Prevalence of hearing loss by severity in the United States. Am J Public Health 2016; 106 (10): 1820-22.

15. Lin FR, Thorpe R, Gordon-Salant S, Ferrucci L. Hearing loss prevalence and risk factors among older adults in the United States. Journals Gerontol - Ser A Biol Sci Med Sci 2011; 66 A (5): 582-90.

16. Zhan W, Cruickshanks KJ, Klein BE, Klein R, Huang GH, Pankow JS, et al. Generational Differences in the Prevalence of Hearing Impairment in Older Adults. Am J Epidemiol. 2010; 171 (2): 260-66.

17. Dubno JR, Lee F-S, Matthews LJ, Ahlstrom JB, Horwitz AR, Mills JH. Longitudinal changes in speech recognition in older persons. J Acoust Soc Am 2008;123 (1): 462-75.

18. Gates GA, Mills JH. Presbycusis. Lancet. 2005; 366: 1111-20.

19. Chen SP, Bhattacharya J, Pershing S. Association of Vision Loss With Cognition in Older Adults. JAMA Ophthalmol 2017; 135 (9): 963.

20. Naël V, Pérès K, Dartigues J-F, Letenneur L, Amieva H, Arleo A, et al. Vision loss and 12-year risk of dementia in older adults: the $3 \mathrm{C}$ cohort study. Eur J Epidemiol 2019; 34 (2): 141-52.

21. Davies-Kershaw HR, Hackett RA, Cadar D, Herbert A, Orrell M, Steptoe A. Vision Impairment and Risk of Dementia: Findings from the English Longitudinal Study of Ageing. J Am Geriatr Soc 2018; 66 (9): 1823-9.

22. Deal JA, Betz J, Yaffe K, Harris T, Purchase-Helzner E, Satterfield S, et al. Hearing impairment and incident dementia and cognitive decline in older adults: The health ABC study. Journals Gerontol - Ser A Biol Sci Med Sci 2017; 72 (5): 703-9.

23. Gallacher J, Ilubaera V, Ben-Shlomo Y, Bayer A, Fish $\mathrm{M}$, Babisch WEP, et al. Auditory threshold, phonologic demand, and incident dementia. Neurology 2012; 79 (15): 1593-90.

24. Gurgel RK, Ward PD, Schwartz S, Norton MC, Foster NL, Tschanz JT. Relationship of Hearing Loss and Dementia. Otol Neurotol 2014; 35 (5): 775-81.

25. Heywood R, Gao Q, Nyunt MSZ, Feng L, Chong MS, Lim WS, et al. Hearing Loss and Risk of Mild Cognitive Impairment and Dementia: Findings from the Singapore Longitudinal Ageing Study. Dement Geriatr Cogn Disord 2017; 43 (5-6): 259-68.

26. Kim SY, Lim J-S, Kong IG, Choi HG. Hearing impairment and the risk of neurodegenerative dementia: A longitudinal follow-up study using a national sample cohort. Sci Rep 2018; 8 (1): 15266. 
27. Lin FR, Metter EJ, O'Brien RJ, Resnick SM, Zonderman $\mathrm{AB}$, Ferrucci L. Hearing Loss and Incident Dementia. Arch Neurol 2011; 68 (2): 214-20.

28. Thomson RS, Auduong P, Miller AT, Gurgel RK. Hearing loss as a risk factor for dementia: A systematic review. Laryngoscope Investig Otolaryngol 2017; 2 (2): 69-79.

29. Lin FR, Yaffe K, Xia J, Xue QL, Harris TB, Purchase-Helzner $\mathrm{E}$, et al. Hearing loss and cognitive decline in older adults. JAMA Intern Med 2013; 173 (4): 293-9.

30. Panza F, Solfrizzi V, Logroscino G. Age-related hearing impairment - A risk factor and frailty marker for dementia and AD. Nat Rev Neurol. 2015; 11 (3): 166-75.

31. Harrison Bush AL, Lister JJ, Lin FR, Betz J, Edwards JD. Peripheral hearing and cognition: Evidence from the staying keen in later life (SKILL) study. Ear Hear 2015; 36 (4): 395-407.

32. Lin FR, Ferrucci L, Metter EJ, An Y, Zonderman AB, Resnick SM. Hearing Loss and Cognition in the Baltimore Longitudinal Study of Aging. Neuropsychology 2011; 25 (6): 763-70.

33. Lin FR, Albert M. Hearing loss and dementia - who is listening? Aging Ment Health 2014; 18 (6): 671-3.

34. Wayne RV, Johnsrude IS. A review of causal mechanis$\mathrm{ms}$ underlying the link between age-related hearing loss and cognitive decline. Ageing Res Rev 2015; 23 (Pt B): 154-66.

35. Peelle JE. Listening effort: How the cognitive consequences of acoustic challenge are reflected in brain and behavior. Ear Hear 2018; 39 (2): 204-14.

36. Alain C, Roye A, Salloum C. Effects of age-related hearing loss and background noise on neuromagnetic activity from auditory cortex. Front Syst Neurosci 2014; 8: 8 .

37. Eckert MA, Cute SL, Vaden KI, Kuchinsky SE, Dubno JR. Auditory cortex signs of age-related hearing loss. J Assoc Res Otolaryngol 2012; 13 (5): 703-13.

38. Engle JR, Tinling S, Recanzone GH. Age-related hearing loss in rhesus monkeys is correlated with cochlear histopathologies. Snyder J, ed. PLoS One 2013; 8 (2): e55092.

39. Hughes LF, Turner JG, Parrish JL, Caspary DM. Processing of broadband stimuli across A1 layers in young and aged rats. Hear Res 2010; 264 (1-2): 79-85.

40. Martin del Campo HN, Measor KR, Razak KA. Parvalbumin immunoreactivity in the auditory cortex of a mouse model of presbycusis. Hear Res 2012; 294 (1-2): 31-9.

41. Peelle JE, Troiani V, Grossman M, Wingfield A. Hearing Loss in Older Adults Affects Neural Systems Supporting Speech Comprehension. J Neurosci 2011; 31 (35): 12638-43.
42. Skoe E, Krizman J, Anderson S, Kraus N. Stability and plasticity of auditory brainstem function across the lifespan. Cereb Cortex 2015; 25 (6): 1415-26.

43. Peelle JE, Wingfield A. The Neural Consequences of Age-Related Hearing Loss. Trends Neurosci 2016; 39 (7): 486-97.

44. Dawes P, Emsley R, Cruickshanks KJ, Moore DR, Fortnum $\mathrm{H}$, Edmonson-Jones $\mathrm{M}$, et al. Hearing Loss and Cognition: The Role of Hearing Aids, Social Isolation and Depression. PLoS One 2015; 10 (3): e0119616.

45. Fratiglioni L, Paillard-Borg S, Winblad B. An active and socially integrated lifestyle in late life might protect against dementia. Lancet Neurol 2004; 3 (6): 343-53.

46. Kuiper JS, Zuidersma M, Oude Voshaar RC, Zuidema SU, van den Heuvel ER, Stolk RP, et al. Social relationships and risk of dementia: A systematic review and meta-analysis of longitudinal cohort studies. Ageing Res Rev 2015; 22: 39-57.

47. Mick P, Kawachi I, Lin FR. The Association between Hearing Loss and Social Isolation in Older Adults. Otolaryngol Neck Surg. 2014; 150 (3): 378-84.

48. Shankar A, Hamer M, McMunn A, Steptoe A. Social Isolation and Loneliness. Psychosom Med 2013; 75 (2): 161-70.

49. Chen DS, Genther DJ, Betz J, Lin FR. Association Between Hearing Impairment and Self-Reported Difficulty in Physical Functioning. J Am Geriatr Soc 2014; 62 (5): 850-6.

50. Mener DJ, Betz J, Genther DJ, Chen D, Lin FR. Hearing Loss and Depression in Older Adults. J Am Geriatr Soc 2013; 61 (9): 1627-9.

51. Park SY, Kim MJ, Sikandaner H, Kim DK, Yeo SW, Park SN. A causal relationship between hearing loss and cognitive impairment. Acta Otolaryngol 2016; 136 (5): 480-3.

52. Park SY, Kim MJ, Kim HL, Kim DK, Yeo SW, Park SN. Cognitive decline and increased hippocampal p-tau expression in mice with hearing loss. Behav Brain Res 2018; 342: 19-26.

53. Belkhiria C, Vergara RC, San Martín S, Leiva A, Marcenaro B, Martínez M, et al. Cingulate Cortex Atrophy Is Associated With Hearing Loss in Presbycusis With Cochlear Amplifier Dysfunction. Front Aging Neurosci 2019; 26 (3): 11: 97.

54. Liu C-M, Lee CT-C. Association of Hearing Loss With Dementia. JAMA Netw open 2019; 2 (7): e198112.

55. Roman GC, Nash DT, Fillit H. Translating current knowledge into dementia prevention. Alzheimer Dis Assoc Disord 2012; 26 (4): 295-9.

56. Kim MB, Zhang Y, Chang Y, Ryu S, Choi Y, Kwon MJ, et al. Diabetes mellitus and the incidence of hearing loss: 
a cohort study. Int J Epidemiol 2017; 46 (2): 717-26.

57. Baumgart M, Snyder HM, Carrillo MC, Fazio S, Kim H, Johns H. Summary of the evidence on modifiable risk factors for cognitive decline and dementia: A population-based perspective. Alzheimer's Dement. 2015; 11 (6): 718-26.

58. Wilson BS, Tucci DL, Merson MH, O’Donoghue GM. Global hearing health care: new findings and perspectives. Lancet. 2017; 390 (10111): 2503-15.

59. Tikka C, Verbeek JH, Kateman E, Morata TC, Dreschler WA, Ferrite S. Interventions to prevent occupational noise-induced hearing loss. Cochrane Database Syst Rev 2017; 7: CD006396.

60. Kuo H-K, Jones RN, Milberg WP, Tennstedt S, Talbot L, Morris JN, et al. Effect of Blood Pressure and Diabetes Mellitus on Cognitive and Physical Functions in Older Adults: A Longitudinal Analysis of the Advanced Cognitive Training for Independent and Vital Elderly Cohort. J Am Geriatr Soc 2005; 53 (7): 1154-61.

61. Yamasoba T, Lin FR, Someya S, Kashio A, Sakamoto T, Kondo K. Current concepts in age-related hearing loss: epidemiology and mechanistic pathways. Hear Res 2013; 303: 30-8.

62. Morris AE, Lutman ME, Cook AJ, Turner D. An economic evaluation of screening 60-to 70-year-old adults for hearing loss. J Public Heal (United Kingdom). 2013; 35 (1): 139-46.

63. Linssen AM, Anteunis LJC, Joore MA. The Cost-Effectiveness of Different Hearing Screening Strategies for 50- to 70-Year-Old Adults: A Markov Model. Value Heal 2015; 18 (5): 560-9.

64. Spiby J. Screening for Hearing Loss in Older Adults: External Review against the Programme Appraisal Criteria for the UK National Screening Committee (UK NSC)[Internet]. 2014 [Consultado el 30 de diciembre de 2018]. Disponible en: http://www.thebsa.org.uk/ wp-content/uploads/2015/09/Hearing_screening_in_ adults_review-NSC.pdf

65. Cardemil MF, Muñoz SD, Fuentes LE. Hipoacusia asociada al envejecimiento en Chile: ¿En qué aspectos se podría avanzar? Rev Otorrinolaringol y cirugía cabeza y cuello 2016; 76 (1): 127-35.

66. Chou R, Dana T, Bougatsos C, Fleming C, Beil T. Screening adults aged 50 years or older for hearing loss: A review of the evidence for the U.S. preventive services task force. Ann Intern Med 2011; 154 (5): 347-55.

67. Raman G, Lee J, Chung M, Gaylor JM, Sen S, Rao M, et al. Effectiveness of Cochlear Implants in Adults with Sensorineural Hearing Loss. Agency for Healthcare Research and Quality (US); 2011 [Consultado el 26 de octubre de 2019]. Disponible en: http://www.ncbi.nlm. nih.gov/pubmed/25927131.

68. Specialised Commissioning Team - NHS. Clinical Commissioning Policy: Bone conducting hearing imlants for hearing loss [Internet]. 2016 [Consultado el 26 de octubre de 2019]. Disponible en: https://www. england.nhs.uk/commissioning/wp-content/uploads/ sites/12/2013/05/16041_FINAL.pdf

69. NHS. Hearing loss - Treatment - NHS [Internet]. 2018 [Consultado el 26 de diciembre de 2018]. Disponible en: https://www.nhs.uk/conditions/hearing-loss/treatment/

70. Regan J, Dawes P, Pye A, Armitage CJ, Hann M, Himmelsbach I, et al. Improving hearing and vision in dementia: Protocol for a field trial of a new intervention. BMJ Open 2017; 7 (11): e018744.

71. Mulrow CD, Aguilar C, Endicott JE, Tuley MR, Velez R, Charlip WS, et al. Quality-of-life changes and hearing impairment. A randomized trial. Ann Intern Med 1990; 113 (3): 188-94.

72. Weinstein BE, Sirow LW, Moser S. Relating Hearing Aid Use to Social and Emotional Loneliness in Older Adults. Am J Audiol 2016; 25 (1): 54.

73. MINSAL. Guía Clínica Hipoacusia Bilateral en personas de 65 años y más que requieren uso de audífono [Internet]. Santiago; 2013 [Consultado el 9 de febrero de 2019]. Disponible en: https://www.minsal.cl/sites/ default/files/files/Hipoacusiabilateralmayores65agnos. pdf.

74. Olusanya OB, Neumann JK, Saunders EJ. The global burden of disabling hearing impairment: a call to action. Bull World Health Organ. [Internet] 2014; 92 (5): 367-73. Disponible en: https://apps.who.int/iris/ handle/10665/271503

75. Dawes P, Cruickshanks KJ, Fischer ME, Klein BEK, Klein R, Nondahl DM. Hearing-aid use and long-term health outcomes: Hearing handicap, mental health, social engagement, cognitive function, physical health, and mortality. Int J Audiol 2015; 54 (11): 838-44.

76. Bisgaard N, Ruf S. Findings From EuroTrak Surveys From 2009 to 2015: Hearing Loss Prevalence, Hearing Aid Adoption, and Benefits of Hearing Aid Use. Am J Audiol 2017; 26 (3S): 451.

77. Chien W, Lin FR. Prevalence of hearing aid use among older adults in the United States. Arch Intern Med 2012; 172 (3): 292-3.

78. Hartley D, Rochtchina E, Newall P, Golding M, Mitchell P. Use of Hearing Aids and Assistive Listening Devices in an Older Australian Population. J Am Acad Audiol 2010; 21 (10): 642-53.

79. Thodi C, Parazzini M, Kramer SE, Davis A, Stenfelt S, Janssen T, et al. Adult hearing screening: Follow-up and outcomes. Am J Audiol 2013; 22 (1): 183-5. 
80. León IA, Ediap RR, Carvallo TR. Adherencia al uso de audífonos en adultos mayores del Servicio de Salud Aconcagua. Rev Otorrinolaringol y cirugía cabeza y cuello 2010; 70 (1): 37-42.

81. Cardemil F, Barría T, Aguayo L, Esquivel P, Rahal M, Fuente A, et al. Evaluación del programa "Active Communication Education" para rehabilitación auditiva en Adultos Mayores con hipoacusia usuarios de audífonos. 2014; 74: 93-100.

82. Mamo SK, Reed NS, Nieman CL, Oh ES, Lin FR. Personal Sound Amplifiers for Adults with Hearing Loss. Am J Med 2016; 129 (3): 245-50.

83. Mamo SK, Nirmalasari O, Nieman CL, McNabney MK, Simpson A, Oh ES, et al. Hearing Care Intervention for Persons with Dementia: A Pilot Study. Am J Geriatr Psychiatry 2017; 25 (1): 91-101.

84. Nieman CL, Marrone N, Mamo SK, Betz J, Choi JS, Contrera KJ, et al. The Baltimore HEARS Pilot Study: An Affordable, Accessible, Community-Delivered Hearing Care Intervention. Gerontologist. 2016; 57 (6): 1173-86.

85. Hoppe U, Hesse G. Hearing aids: indications, technology, adaptation, and quality control. GMS Curr Top Otorhinolaryngol Head Neck Surg. 2017; 16 (12): Doc 08.

86. Kalluri S, Humes LE. Hearing technology and cognition. Am J Audiol. 2012; 21 (2): 338-43.

87. Maharani A, Dawes P, Nazroo J, Tampubolon G, Pendleton N, Bertelsen G, et al. Longitudinal Relationship Between Hearing Aid Use and Cognitive Function in Older Americans. J Am Geriatr Soc. 2018; 66 (6): 11306.

88. Dawes P, Wolski L, Himmelsbach I, Regan J, Leroi I. Interventions for hearing and vision impairment to improve outcomes for people with dementia: A scoping review. International Psychogeriatrics. 2019; 31 (2): 20321.

89. Volter C, Gotze L, Dazert S, Thomas JP, Falkestein M. Can cochlear implantation improve neurocognition in the aging population? Clin Interv Aging. 2018; 13 (4): 701-12.

90. Moyer VA. Screening for hearing loss in older adults: U.S. preventive services task force recommendation statement. Ann Intern Med 2012; 157 (9): 655-61.
91. Yueh B, Shapiro N, MacLean CH, Shekelle PG. Screening and Management of Adult Hearing Loss in Primary Care: Scientific Review. J Am Med Assoc 2003; 289 (15): 1976-85.

92. Davis A, Smith P, Ferguson M, Stephens D. Acceptability, benefit and costs of early screening for hearing disability. Heal Technol Assess NHS R\&D HTA Program 2007; 11 (42): 1-293.

93. Mick P, Pichora-Fuller MK. Is Hearing Loss Associated with Poorer Health in Older Adults Who Might Benefit from Hearing Screening? Ear Hear 2016; 37 (3): e194-e201.

94. Ventry IM, Weinstein BE. The hearing handicap inventory for the elderly: A new tool. Ear Hear 1982; 3 (3): 128-34.

95. Labos E, Slachevsky A, Torralva T, Fuentes P. MF. Tratado de Neuropsicología Clínica del Adulto. Segunda Edición. Buenos Aires, Argentina: Librería Akadia; 2018.

96. Jorgensen LE, Palmer CV, Pratt S, Erickson KI, Moncrieff D. The Effect of Decreased Audibility on MMSE Performance: A Measure Commonly Used for Diagnosing Dementia. J Am Acad Audiol 2016; 27 (4): 311-23.

97. Uhlmann RF, Teri L, Rees TS, Mozlowski KJ, Larson EB. Impact of mild to moderate hearing loss on mental status testing. Comparability of standard and written Mini-Mental State Examinations. J Am Geriatr Soc 1989; 37 (3): 223-8.

98. Recommendations | Hearing loss in adults: assessment and management | Guidance | NICE [Internet]. NICE; [Consultado el 25 de octubre de 2019]. Disponible en: https://www.nice.org.uk/guidance/ng98/chapter/ Recommendations\#adults-with-suspected-or-diagnosed-dementia-mild-cognitive-impairment-or-a-learning-disability

99. Care CTF on PH, Pottie K, Rahal R, Jaramillo A, Birtwhistle R, Thombs BD, et al. Recommendations on screening for cognitive impairment in older adults. C Can Med Assoc J 2016; 188 (1): 37.

100. Falk N, Cole A, Meredith TJ. Evaluation of Suspected Dementia. Am Fam Physician 2018; 97 (6): 398-405.

101. Quiroga LP, Albala BC, Klaasen PG. Validación de un test de tamizaje para el diagnóstico de demencia asociada a edad, en Chile. Rev Med Chile 2004; 132 (4): 467-78. 